\title{
Spezialisierte Behindertenmedizin?
}

\author{
Felix Brem \\ Dr. med., Mitglied FMH
}

\section{Einleitung}

Vor zwei Jahren trat die UNO-Behindertenrechtskonvention (BRK) in der Schweiz in Kraft. Welche Konsequenzen hat dieses Vertragswerk auf die medizinische Behandlung und die Versorgungsstrukturen? An einem von der Schweizerischen Arbeitsgemeinschaft von Ärzten für Menschen mit geistiger oder mehrfacher Behinderung (SAGB) in Zusammenarbeit mit der Fachhochschule Nordwestschweiz einberufenen Kick-off-Hearing am 22.4.2016 in Olten kamen Menschen mit Behinderung, Angehörige und Fachleute zu Wort. Im Mittelpunkt standen die besonders benachteiligten Gruppen der Menschen mit AutismusStörungen, Zerebralparesen oder sogenannt seltener Krankheiten. Angesprochen waren aber alle Menschen mit irgendeiner Behinderung, also deutlich mehr als 10\% der Bevölkerung. Die Tagung wurde von der FMH und Curafutura wohlwollend unterstützt.

Die medizinische Versorgung für Menschen mit einer Behinderung in der Schweiz ist insgesamt gut, doch in mehreren Bereichen gibt es gravierende Lücken insbesondere für Erwachsene, wurde einleitend festgehal-

\section{Résumé}

Les soins médicaux sous l'angle de la convention de I'ONU relative aux droits des personnes avec handicap

II y a deux ans que la convention de I'ONU relative aux droits des personnes avec handicap (CDPH) est entrée en vigueur en Suisse. Quelles sont les conséquences de ce traité sur les soins médicaux et sur les structures de soin? À l'occasion d'une audition convoquée par I'Association suisse de médecins s'occupant de personnes avec handicap mental ou polyhandicap (ASHM) à Olten, la parole a été donnée aux personnes avec handicap, à leurs proches et aux professionnels.

La prise en charge des personnes avec handicap en Suisse est bonne dans l'ensemble, mais l'on constate néanmoins de graves lacunes dans plusieurs domaines. En effet, les témoignages des patients et des proches montrent clairement que I'article 25 (CDPH), qui exige des parties contractantes des soins de base de même qualité et selon les mêmes normes pour les personnes avec handicap que pour les autres personnes, n'est toujours pas appliqué de manière satisfaisante.

Selon Caroline Hess-Klein d'Inclusion Handicap, I'association faîtière des organisations de personnes avec handicap, la Suisse est obligée d'appliquer la CDPH-ONU. D'ici la mi-mai 2016, la Suisse doit soumettre à l'ONU un rapport sur l'état de la mise en œuvre.

Les personnes avec handicap ont besoin de temps, de compréhension et de patience. Elles représentent un défi pour un système de plus en plus axé sur des critères standardisés, sur I'efficacité et la rentabilité. Selon le conseiller national Christian Lohr, une attitude de partenariat fondée sur le dialogue entre les patients et le personnel soignant est la condition de base pour garantir le succès d'un traitement. ten. Der Hilferuf eines verzweifelten Jugendlichen mit frühkindlichem Autismus als einleitendes Beispiel offenbarte seine unerkannte grosse Not. Die Erfahrungsberichte von Betroffenen und Angehörigen machten tatsächlich deutlich, dass Art. 25 BRK, wonach die Vertragsparteien Menschen mit Behinderungen eine Gesundheitsversorgung in derselben Bandbreite, von derselben Qualität und auf demselben Standard wie anderen Menschen zur Verfügung stellen sollen, und insbesondere auch Gesundheitsleistungen anbieten, die von Menschen mit Behinderungen speziell wegen ihrer Behinderungen benötigt werden, noch der Umsetzung harrt. Es braucht spezialisiertes Wissen, spezielle kommunikative Kompetenzen und Erfahrungen und geeignete Rahmenbedingungen zur Verbesserung der Versorgungssituation.

\section{Die UN-Behindertenrechtskonvention}

Das am 15. Mai 2014 in der Schweiz in Kraft getretene Vertragswerk, das zusammen mit Menschen mit Behinderung erarbeitet wurde, haben bis heute 163 Staaten ratifiziert. Die Schweiz habe sich mit der Ratifizierung verpflichtet, die UNO-BRK umzusetzen, betonte Frau lic. iur. Caroline Hess-Klein von Inclusion Handicap, dem Dachverband der Behindertenorganisationen. Bis Mitte 2016 muss die Schweiz der UNO einen ersten Bericht über den Stand der Umsetzung unterbreiten, danach alle vier Jahre.

Mittels der Austauschplattform "Sensor UNO-BRK» unter www.uno-brk.ch erhebt Inclusion Handicap derzeit, mit welchen Problemen und Hindernissen Menschen mit Behinderungen konfrontiert sind. Die Resultate werden in den sogenannten Schattenbericht einfliessen, den Inclusion Handicap Anfang 2017 bei der UNO als Stimme der Zivilgesellschaft einreichen wird. Doch bereits jetzt könnten Missstände benannt werden, sagte Hess-Klein: Viel zu oft würden andere anstelle der betroffenen Personen über die medizinische Behandlung entschieden; was nicht der in der UNO-BRK verankerten Selbstbestimmung entspreche. Auch bezüglich einer angemessenen Versorgung von Menschen mit Behinderung würden zum Teil erhebliche Mängel rapportiert. 




Die medizinische Versorgung für Menschen mit einer Behinderung in der Schweiz ist insgesamt gut, doch in mehreren Bereichen gibt es gravierende Lücken. So sagt Yvonne Hämmig, die mit einer zerebralen Bewegungsbehinderung auf die Welt gekommen war, über ihren kürzlichen Spitalaufenthalt: «Ich fühlte mich als Mensch mit einer Bewegungsbehinderung weitgehend nicht ernst genommen, in meiner Würde verletzt und ausgeliefert.»

\section{Zu wenig Kommunikation, zu wenig Information}

Yvonne Hämmig, die mit einer zerebralen Bewegungsbehinderung auf die Welt gekommen war, verdeutlichte anhand eines kürzlichen Spitalaufenthaltes, woran das System krankt: «Viele Abläufe wären vereinfacht worden, wenn man mir etwas genauer zugehört hätte», so die Bilanz der ausgebildeten Sozialarbeiterin. «Ich fühlte mich als Mensch mit einer Bewegungsbehinderung weitgehend nicht ernst genommen, in meiner Würde verletzt und ausgeliefert. Ich denke, dass ich als Patientin ein Anrecht auf Anhörung sowie genügende Informationen gehabt hätte.»

Nur interprofessionell können die oft komplexen Fragestellungen in der Behindertenmedizin erfolgreich angegangen werden.

Serge Viel, Vater eines 21-jährigen Sohnes ohne Lautsprache, mit Zerebralparese und mehrfacher körperlicher Behinderung, wies ebenso auf Missstände in einem von Kosteneffizienz und standardisierten Abläufen geprägten System hin: Extrem lange Wartezeiten bei Untersuchungen, fehlende Anhörung der erfahrenen Eltern wie auch das Unvermögen von Fachleuten im Spital im Umgang mit Menschen, die nicht sprechen können, werden Menschen mit besonderen Bedürfnissen nicht gerecht.

\section{Frühere Entlassungen aufgrund der SwissDRG}

Auch das Anfang 2012 schweizweit eingeführte Tarifsystem für stationäre Spitalleistungen nach Fallpauschalen (SwissDRG) hat zum Teil gravierende Konsequenzen: «Unsere Klienten müssen das Spital heute sehr früh wieder verlassen", stellte Sonja Weth fest, Leiterin Fachdienst Gesundheit bei abilia, einem Verein, der in Basel unterschiedliche Betreuungssettings für Menschen mit kognitiver Beeinträchtigung anbietet. Mit der Einführung obligatorischer Eintritts- und Austrittsgespräche zwischen den Fachleuten der Institution und den Verantwortlichen im Spital zur ausführlichen Information und zur Klärung der medizinischen und pflegerischen Möglichkeiten in der Institution konnte die Situation deutlich entspannt werden; auch die Gesprächspartner von Spitalseite würden zurückmelden, dass ihnen dieses Setting hilft, um zu einer klaren Einschätzung zu kommen.

\section{Lücken in der Aus- und Weiterbildung}

Florian Suter, der als Allgemeinpraktiker über langjährige Erfahrung in der Versorgung von Menschen mit Behinderung verfügt, ortet vor allem in der Aus- und Weiterbildung von Gesundheitsfachpersonen Handlungsbedarf: «Menschen mit Behinderung werden heute im Ausbildungsparcours kaum thematisiert.» Ärztliche Betreuung von Menschen mit Behinderungen ist anforderungsreich, befriedigend, dankbar und wäre es unbedingt wert, in der Ausbildung oder Weiterbildung konsequent vermittelt werden. Notwendig sei aber ebenso die Offenheit der Fachkräfte für nicht klassische Vorgehensweisen, so sein Appell. Er wies zudem auf die Aktivitäten der SAMW bezüglich professioneller Zusammenarbeit hin. Nur interprofessionell können die oft komplexen Fragestellungen in der Behindertenmedizin erfolgreich angegangen werden.

\section{Mettre en réseau, sensibiliser, informer}

L'Association suisse de médecins s'occupant de personnes avec handicap mental ou polyhandicap (ASHM) s'engage pour une amélioration de la prise en charge médicale et de la qualité de vie des personnes avec handicap mental ou polyhandicap. Fondée en 2008, cette société a pour but de mettre en relation tous les médecins prenant soin de ces patients, de sensibiliser le corps médical à cette thématique et de créer un programme de formation continue. 


\section{Seltene Erkrankungen}

Gert Printzen, ehemaliges Mitglied des Zentralvorstands FMH und deren Delegierter in der IG seltene Krankheiten (IGSK), pflichtete ergänzend bei: Es brauche bereits während der Ausbildung mehr Sensibilisierung für komplexe Krankheitsbilder und heterogene Gruppen, die in Diagnose und Behandlung anspruchsvoll sind. Die Seltenheit der einzelnen Erkrankungen erschwert aus medizinischen und ökonomischen Gründen häufig die Forschung und die medizinische

Die Medizin konzentriert sich häufig allzu einseitig auf Professionalität; Menschlichkeit und der gesunde Menschenverstand gehen dabei manchmal vergessen.

Versorgung der betroffenen Patientinnen und Patienten, die oft die Diagnose verzögert erhalten und sich alleine gelassen fühlen. Es fehlt an Experten. Durch entsprechende Richtlinien müssten der Versorgungsbereich der ambulanten spezialfachärztlichen Versorgung näher ausgestaltet und weitere Regelungen u.a. zur Konkretisierung der Erkrankungen und des Behandlungsumfangs, zu sächlichen und personellen Anforderungen an die Leistungserbringung sowie zu sonstigen Anforderungen an die Qualitätssicherung getroffen werden. Um die Situation zu verbessern, ist die Erforschung von diagnostischen und therapeutischen Massnahmen für seltene Erkrankungen weiter zu fördern. Ergänzend zur medizinischen Versorgung stellt die Selbsthilfe immer mehr eine zentrale Säule im Gesundheitssystem dar.

\section{Behandlungen auf Augenhöhe}

Korrespondenz: Dr. med. Felix Brem Rathausstrasse 17 CH-8570 Weinfelden Felix.Brem[at]hin.ch

Die Podiumsdiskussion mit Betroffenen und Fachpersonen aus Medizin und Behindertenverbänden machte erneut deutlich: Menschen mit Behinderung brauchen Verständnis, Zeit und Geduld, und sie sind eine Herausforderung für ein System, das sich an standardisierten Messkriterien, Effizienz und Wirtschaftlichkeit orientiert. Doch ist gerade eine partnerschaftliche, dialogorientierte Haltung zwischen Patienten und Behandelnden eine Grundvoraussetzung für eine erfolgreiche Behandlung.

«Wer nicht zuhört, hat auch kein Recht zu reden», resümierte Nationalrat Christian Lohr. Bei der medizinischen Behandlung von Menschen mit Behinderung ginge es letztlich um eine Grundhaltung; um die Frage des Umgangs miteinander. «Menschen müssen als gleichwertig angesehen werden", so sein Fazit, das er auch gesundheitspolitisch verstanden wissen will.

\section{Schlussbemerkungen}

Die Medizin konzentriert sich häufig allzu einseitig auf Professionalität; Menschlichkeit und der gesunde Menschenverstand gehen dabei manchmal vergessen. Betroffene müssen ihre Forderungen äussern, die erforderlichen Veränderungen jedoch müssen von der Politik gewollt sein und vom ganzen Behandlungssystem getragen werden. Eine Arbeitsgruppe der SAGB will sich nun weiter mit dem Thema befassen. Drei Problemkreise stehen vorerst im Vordergrund:

- Konsequenzen für die Aus-/Weiter-/Fortbildung verschiedener Berufe, insbesondere aber der Ärzte.

- Spezifische Unterstützungsangebote für die Kommunikation und die Gesundheitsvorsorge Betroffener

- Spezielle Kompetenzzentren in Ergänzung zur hausund spezialärztlichen Versorgung, zu den Spitälern und Universitätsinstituten

Bildnachweis

c) Vladkol | Dreamstime.com
Weitere Informationen, insbesondere die Referate und Literaturhinweise, finden sich unter www.sagb.ch $\rightarrow$ Veranstaltungen $\rightarrow$ Hearing Behindertenmedizin sowie $\rightarrow$ Literatur zum 22.4.2016. 